\title{
Elaboración de una escala para el análisis de los valores adquiridos con el Modelo de Responsabilidad Personal y Social en el contexto escolar (ECVA-12)
}

\section{Development of a scale for the analysis of the values acquired with the Personal and Social Responsibility Model in scholar setting (ECVA-12)}

\section{Desenvolvimento de uma escala para análise dos valores adquiridos com o Modelo de Responsabilidade Pessoal e Social em contexto escolar (ECVA-12)}

\author{
Rodríguez, I. ${ }^{1}$, Valero-Valenzuela, A. ${ }^{1}$, Manzano-Sánchez, D. ${ }^{1}$ \\ ${ }^{1}$ Universidad de Murcia, Campus de San Javier, España
}

\begin{abstract}
RESUMEN
El objetivo de la presente investigación fue el diseño y la validación de una escala que permitiera analizar los valores adquiridos con el Modelo de Responsabilidad Personal y Social en escolares, en comparación con la metodología impartida previamente por el docente (ECVA-12) desde las diferentes materias del currículum escolar. El estudio de carácter transversal y descriptivo se llevó a cabo en varias fases y con muestras diferentes: con una muestra de 104 estudiantes entre 9 y 15 años, validez de contenido mediante la técnica Delphi con 11 jueces expertos, validez de comprensión, análisis de la estabilidad temporal y validez de constructo mediante Análisis Factorial Exploratorio. Posteriormente, y en este caso con una muestra de 76 estudiantes de entre 12 y 15 años, se realizó un Análisis Factorial Confirmatorio, mostrando valores de ajuste adecuados en un modelo de 2 factores y 12 ítems, con saturaciones e índices de consistencia interna elevados tanto en escala como en las sub-escalas. Los resultados obtenidos confirman que el instrumento diseñado cumple con los criterios de validez y fiabilidad establecidos, capaz de medir el grado de satisfacción y los cambios percibidos en el comportamiento de los estudiantes, convirtiéndolo en una herramienta básica para investigaciones relacionadas con la aplicación de este modelo de enseñanza.
\end{abstract}

Palabras clave: educación, instrumento, validación, metodología, MRPS.

\begin{abstract}
The objective of this research was the design and validation of a scale that would allow the analysis of the values acquired with the Model of Personal and Social Responsibility in schoolchildren, in comparison with the methodology previously taught by the teacher (ECVA-12) from the different subjects of the school curriculum. The cross-sectional and descriptive study was carried out in several phases and with different samples: with a sample of 104 students between 9 and 15 years old, content validity using the Delphi technique with 11 expert judges, comprehension validity, analysis of the temporal stability and construct validity through Exploratory Factor Analysis. Subsequently, and in this case with a sample of 76 students between 12 and 15 years old, a Confirmatory Factor Analysis was performed, showing adequate adjustment values in a 2-factor and 12-item model, with saturations and high internal consistency indices both in scale as in the subscales. The results obtained confirm that the designed
\end{abstract}




\section{Medida de la Responsabilidad Personal y Social en contexto escolar}

instrument meets the established validity and reliability criteria, capable of measuring the degree of satisfaction and the perceived changes in the behavior of students, making it a basic tool for research related to the application of this teaching model.

Keywords: education, tool, validation, methodology, TPSR.

\section{RESUMO}

O objetivo desta pesquisa foi o desenho e validação de uma escala que permitisse a análise dos valores adquiridos com o Modelo de Responsabilidade Pessoal e Social em escolares, em comparação com a metodologia previamente ensinada pelo professor (ECVA-12) das diferentes disciplinas do currículo escolar. O estudo transversal e descritivo foi realizado em várias fases e com amostras distintas: com amostra de 104 alunos entre 9 e 15 anos, validade de conteúdo pela técnica Delphi com 11 juízes especialistas, validade de compreensão, análise de estabilidade temporal e validade de construto por meio da Análise Fatorial Exploratória. Posteriormente, e neste caso com uma amostra de 76 alunos entre 12 e 15 anos, foi realizada uma Análise Fatorial Confirmatória, mostrando valores de ajuste adequados em um modelo de 2 fatores e 12 itens, com saturações e altos índices de consistência interna ambos em escala como nas subescalas. Os resultados obtidos confirmam que o instrumento elaborado atende aos critérios de validade e confiabilidade estabelecidos, capazes de medir o grau de satisfação e as mudanças percebidas no comportamento dos alunos, tornando-se uma ferramenta básica para pesquisas relacionadas à aplicação deste modelo.

Palavras chave: educação, instrumento, validação, metodología, MRPS.

\section{INTRODUCCIÓN}

Atendiendo a los problemas de convivencia que actualmente se dan entre la población joven (Albaladejo-Blázquez, et al., 2013), surge la necesidad de dar respuesta a este problema que se encuentra en el foco de diversos estudios. En España, se puede ver como aspectos como la violencia, la xenofobia, la delincuencia o los problemas en las relaciones interpersonales, tienen al alza, siendo la violencia escolar uno de los tipos de violencia que reflejan la descomposición de nuestra sociedad (AyalaCarrillo, 2015).

Siguiendo a Menéndez-Santurio y Fernández-Río (2017), esta violencia escolar es un problema recurrente que tiene un gran impacto en el ámbito educativo. Si bien, las clases de Educación Física, pueden ser contextos idóneos para poder trabajar este tipo de aspectos, siendo fundamental en estas clases, trabajar con programas educativos que permitan a su vez, crear climas de implicación hacia la tarea y centrados en el alumnado para prevenir problemas de violencia escolar y discriminación de género (Menéndez-Santurio y Fernández-Río, 2017). Todo ello, es debido fundamentalmente a que en estos entornos educativos es más sencillo el acceso a los adolescentes, además de que la variedad y popularidad de estas actividades las hacen muy atractivas para gran parte de las personas en edad escolar y las características de estas actividades permiten que los jóvenes puedan establecer relaciones interpersonales muy abiertas con el educador y entre sus iguales (Sánchez-Alcaraz et al., 2016). Sin embargo, para que surjan cambios en estas conductas y se adquieran valores y actitudes personal y socialmente adecuados, es necesario un trabajo sistemático y con una orientación educativa en el propio contexto deportivo.

El Modelo de Responsabilidad Personal y Social (MRPS) de Hellison (1985) se ha aplicado ampliamente en programas extraescolares, campamentos de verano y en clases de Educación Física y, debido al atractivo práctico que le caracteriza y al hecho de que su pedagogía no está necesariamente vinculada al contenido de actividad física, se ha propuesto que este modelo proporcione un marco eficaz para promover el aprendizaje social y emocional en el currículo escolar (Gordon et al., 2016).

Por otro lado, diversos planteamientos, tales como la aplicación del modelo en otras materias, ha sido abordado en los últimos años. Así, en el estudio de Escartí et al. (2018), se valoró la fidelidad de la implementación por parte de un maestro y el comportamiento de los estudiantes en la Educación Física y en otras áreas temáticas. Los resultados indicaron que el MRPS puede proporcionar un marco efectivo para promover la 


\section{Rodríguez, I.; Valero-Valenzuela, A.; Manzano-Sánchez, D.}

responsabilidad en el currículo escolar. Del mismo modo, son varios los estudios que han comprobado como esta metodología, puede ser útil para la mejora de los valores de responsabilidad, motivación, satisfacción de las necesidades psicológicas básicas o el clima escolar entre otras variables (Manzano-Sánchez y Valero-Valenzuela, 2019; 2020) con resultados superiores incluso comparando su aplicación en todo el currículum que solo en Educación Física (Manzano-Sánchez et al., 2021). Finalmente, destacar que esta metodología también se ha ampliado en la vertiente de la hibridación con otros modelos pedagógicos con resultados muy satisfactorios, por ejemplo, con el Aprendizaje Cooperativo (Fernández y Méndez, 2016), el Modelo de Educación Deportiva (Méndez y Fernández, 2016) o la gamificación (MeleroCañas et al., 2021), así como en el ámbito competitivo (Carreres-Ponsoda et al., 2021). Finalmente indicar, que no solo en el ámbito nacional se está ampliando la muestra de estudios, sino que ya desde hace años, el modelo se ha ampliado aplicándose en países como en Taiwán, comenzando con Pan y Keh (2014), con muy buenos resultados respecto a la mejora del carácter de los estudiantes y el comportamiento social.

Es especialmente importante construir instrumentos válidos que sirvan para poder valorar los efectos de cualquier programa educativo con el fin de conocer si se han producido los efectos esperados.

A su vez, los resultados del MRPS han sido verificados utilizando un cuestionario para valorar los niveles de responsabilidad (Escartí et al. 2011; 2015), así como ver la fidelidad de la intervención, mediante el instrumento TARE diseñado originalmente por Wright y Craig (2011). Por otro lado, el análisis cualitativo también se ha utilizado, tanto mediante metodología observacional (Camerino et al. 2020) o análisis de entrevistas (Manzano-Sánchez et al. 2020).

Si bien, el MRPS profundiza en gran medida en la importancia del fomento de valores educativos concretos, como son el respeto, el esfuerzo, la autonomía o la ayuda a los demás entre otros, como elemento diferenciador de otras metodologías, no existiendo ningún instrumento validado que pueda analizar estos aspectos desarrollados aplicando el MRPS ni tampoco la satisfacción con esta metodología, siendo de destacar estudios como el de Cabezón et al (2018) donde en su cuestionario valoraron la incidencia de la competición en valores como la autonomía y también la satisfacción con la práctica deportiva, pero no encontrándose investigaciones en el ámbito educativo ni en la valoración de la implementación de modelos pedagógicos.

Por tanto, los objetivos de este estudio son diseñar y validar un cuestionario que permita comparar la metodología previa empleada por el docente con el MRPS (ECVA-12), midiendo el grado de satisfacción de los estudiantes y los cambios generados en su comportamiento, especialmente los relacionados con los valores adquiridos con su implementación en las diferentes materias del currículo.

\section{MATERIAL Y MÉTODOS}

\section{Diseño}

La presente investigación es de carácter transversal y descriptiva destinada a diseñar y validar un instrumento que permita conocer el grado de satisfacción y los logros alcanzados tras la implementación del MRPS en escolares y en comparación con la metodología empleada previamente por el profesor, para lo que se recurre a una metodología de tipo cuantitativo.

\section{Participantes}

Para el primer paso, el cual, consistió en el estudio de la validez de comprensión, el análisis de la fiabilidad y el análisis factorial exploratorio, se empleó una muestra de 104 alumnos (53 chicos y 51 chicas) de entre 9 y 15 años. Por otro lado, para el análisis factorial exploratorio se contó con otra muestra compuesta por 76 estudiantes ( 39 chicos y 36 chicas) de entre 12 y 15 años. Con todos los alumnos, se participó en una investigación donde se implementó el MRPS desde las diferentes materias del currículum educativo.

\section{Instrumentos}

El cuestionario creado para este estudio consta de una introducción inicial donde se justifica la investigación y se explican las instrucciones para responder, garantizando el anonimato del participante. Posteriormente aparecían inicialmente 40 ítems expresados como 


\section{Medida de la Responsabilidad Personal y Social en contexto escolar}

afirmaciones que hacen referencia al grado de satisfacción del alumno con el MRPS y a los logros personales alcanzados (capacidades y valores) gracias a la aplicación de este modelo, los cuales se valoran a través de una escala tipo Likert de 5 categorías desde 1 (totalmente en desacuerdo) hasta 5 (totalmente de acuerdo). El cuestionario definitivo, tuvo un total de 12 ítems, con dos dimensiones que hacían referencia a la satisfacción con la metodología empleada y por otro lado, a la promoción de valores. Finalmente, se indica una serie de agradecimientos por la participación.

\section{Procedimiento}

El procedimiento que se llevó a cabo incluyó las siguientes fases (figura 1):

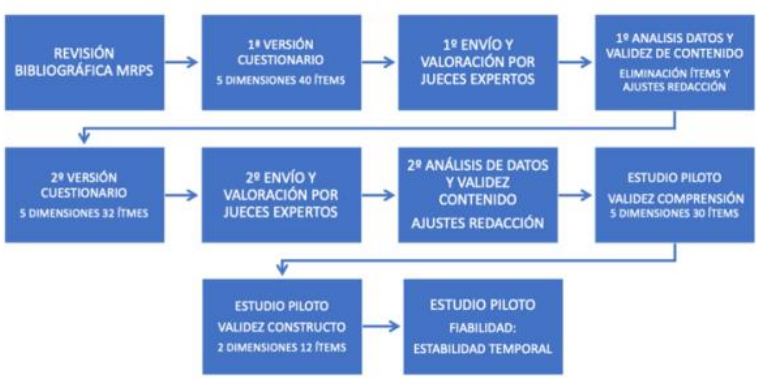

Figura 1. Fases del proceso de construcción y validación del Cuestionario ECVA-12 (Elaboración propia).

Revisión bibliográfica en diferentes bases de datos, como Dialnet, Scopus, y PubMed, con el fin de buscar información sobre las aplicaciones del MRPS y el diseño de herramientas para su evaluación.

Primera versión del cuestionario. Se redactaron 40 ítems relacionados con los niveles del MRPS y con el grado de satisfacción y de descontento en comparación con la metodología que empleaba el profesor anteriormente. Estos ítems fueron agrupados en torno a 5 dimensiones (aspectos positivos y negativos de la nueva metodología, valores sociales alcanzados, valores personales alcanzados y logros generales). En este momento, se puso el investigador principal en contacto con los dos centros, donde se estaba aplicando el Modelo de Responsabilidad Personal.
Primer envío y valoración por jueces expertos. Para ello, el cuestionario fue enviado por correo electrónico a los dos grupos de jueces expertos, considerándose como expertos personal docente en activo o con una experiencia investigadora superior a 10 años en el ámbito educativo (en total, 3 docentes de Educación Primaria, 5 docentes de Educación Secundaria, 3 de Universidad), los cuales, valoraron los cuestionarios de forma cuantitativa y cualitativamente con respecto a los criterios marcados y descritos en una escala de 10 puntos, siguiendo el método Delphi (ReguantÁlvarez y Torrado-Fonseca, 2016).

Primer análisis y validez de contenido. Se llevó a cabo el análisis de las aportaciones de los jueces expertos, lo que sirvió para tomar una serie de decisiones sobre la primera versión del cuestionario. Para ello, además se calculó el índice de Osterlind (1989) y la V de Aiken (Merino y Livia, 2009) y, a pesar de que los resultados fueron óptimos para mantener todos los ítems, se optó por atender a las modificaciones sugeridas y se redujo el número de ítems a 32 (4 por cada dimensión).

Segunda versión del cuestionario. Atendidas todas las aportaciones y valoraciones tanto cuantitativas como cualitativas que realizaron en una primera valoración del cuestionario original, se procedió a recoger todas las decisiones tomadas en una nueva versión del cuestionario, que fue de nuevo enviado a los jueces expertos.

Segundo envío y valoración por jueces expertos. Se analizaron las valoraciones de los expertos y se ejecutaron nuevas modificaciones en cuanto a la redacción y precisión de algunos ítems. Las valoraciones cualitativas sirvieron para interpretar y modificar los ítems que marcaron. En cuanto a las valoraciones cuantitativas, se realizó la prueba V de Aiken (Merino y Livia, 2009) y se optó por mantener los 32 ítems al analizar la valoración media individual de cada ítem por parte de todos los jueces expertos y no obtener ninguno de ellos una valoración inferior a ,70.

Estudio piloto: validez de comprensión. Habiendo adquirido el visto bueno del comité de ética de la Universidad de Murcia (1685/2017), se realizó un estudio piloto con una muestra de estudiantes de Primaria y Secundaria en dos centros educativos 


\section{Rodríguez, I.; Valero-Valenzuela, A.; Manzano-Sánchez, D.}

de similar perfil socio-económico (medio-bajo), para probar la validez de comprensión del cuestionario definitivo. Los alumnos completaron el cuestionario y valoraron, en una escala de 10 puntos, su comprensión global. El cuestionario se pasó en un ambiente tranquilo, tras explicar las instrucciones, empleando entre 8 y 12' para su contestación, siendo supervisado por un miembro del equipo investigador y el docente de la asignatura o área que se estuviera impartiendo, donde se aplicaba el MRPS. La recogida de datos se realizó en enero de 2018.

Estudio piloto: validez de constructo. Esta fase supuso el estudio de la dimensionalidad del instrumento mediante un Análisis Factorial Exploratorio (AFE) con un total de 104 participantes para determinar la validez de constructo del instrumento y un Análisis Factorial Confirmatorio (AFC) para determinar la validez de constructo del instrumento con un total de 76 participantes. La recogida de datos se realizó en marzo de 2018.

Estudio piloto: fiabilidad. Pasadas tres semanas desde el primer estudio, los estudiantes volvieron a responder el cuestionario para comprobar la estabilidad temporal mediante la prueba test-retest y garantizar así la fiabilidad del cuestionario definitivo.

\section{Análisis estadístico}

Para el tratamiento de los datos se utilizó el programa SPSS 24, llevando a cabo diferentes técnicas de análisis de datos. Se analizó la frecuencia de los 32 ítems para comprobar la existencia de valores perdidos y se llevó a cabo la detección de casos atípicos y el cálculo de la Distancia de Mahalanobis, así como los índices de asimetría y curtosis. La consistencia interna se obtuvo a través del Alfa de Cronbach, factor de correlación corregido ítem-total (CCIT-c). Además, se estudiaron las correlaciones bivariadas, se realizó el Análisis Factorial Exploratorio para determinar la validez de constructo y un Análisis Factorial Confirmatorio para confirmar las propiedades psicométricas del modelo propuesto. Se calcularon los índices CFI, NFI, SRMR y RMSEA con sus intervalos de confianza (CI: 90\%). Para estos índices, se consideraron como valores aceptables CFI y NFI $>0.90$, SRMR y RMSEA $<0.08$, siguiendo las recomendaciones de varios autores (Hair et al., 2014; Byrne, 2010; Marsh et al., 2004). La fiabilidad de la escala final se obtuvo mediante el Alfa de Cronbach y el Test-Retest.

\section{RESULTADOS}

\section{Validez de contenido}

Tanto en la primera como segunda versión de la escala se realizaron las pruebas de Osterlind (1989) y la V de Aiken (Merino y Livia, 2009), para obtener la valoración de los jueces expertos sobre cada uno de los 40 ítems que componían la primera versión del cuestionario. Las valoraciones medias atendiendo a los tres criterios y las individuales atendiendo a cada ítem cumplieron las condiciones para ser mantenidos en el cuestionario, aún así se llevaron las modificaciones atendiendo a las consideraciones cualitativas de los expertos.

En la tabla 1 se refleja las valoraciones medias de los jueces expertos atendiendo al contenido y al formato del cuestionario global. Las puntuaciones fueron altas, sin embargo, se optó por seguir depurando el cuestionario aplicando las propuestas de mejora de los jueces expertos y se eliminaron 8 ítems.

\begin{tabular}{|c|c|c|c|}
\hline & IC 95\% & $\begin{array}{l}\mathrm{V} \text { de } \\
\text { Aiken } \\
\end{array}$ & Osterlind \\
\hline $\begin{array}{l}\text { A. En cuanto al } \\
\text { contenido }\end{array}$ & & & 9.24 \\
\hline $\begin{array}{l}\text { Pertinencia de las } \\
\text { dimensiones. }\end{array}$ & $(.830-.943)$ & .90 & 9.45 \\
\hline $\begin{array}{l}\text { Equilibrio entre } \\
\text { dimensiones. }\end{array}$ & $(.830-.943)$ & .90 & 9.45 \\
\hline $\begin{array}{l}\text { B. En cuanto al } \\
\text { formato }\end{array}$ & & & 8.64 \\
\hline $\begin{array}{l}\text { Adecuación de la } \\
\text { redacción de los } \\
\text { ítems a la edad de la } \\
\text { muestra. }\end{array}$ & $(.716-.864)$ & .80 & 8.55 \\
\hline $\begin{array}{lll}\text { Claridad } & \text { en } & \text { la } \\
\text { redacción } & \text { de } & \text { los } \\
\text { ítems } & & \end{array}$ & $(.716-.864)$ & .80 & 8.09 \\
\hline $\begin{array}{l}\text { Organización de los } \\
\text { ítems }\end{array}$ & $(.830-.943)$ & .90 & 9.27 \\
\hline
\end{tabular}




\section{Medida de la Responsabilidad Personal y Social en contexto escolar}

\section{Validez de comprensión}

De los 104 participantes que cumplimentaron la tabla para valorar el cuestionario en general, ninguno realizó preguntas sobre el cuestionario en el apartado destinado para tal fin y la valoración media fue de 8.73 .

\section{Consistencia interna de la escala inicial.}

Definidos de forma exhaustiva y clara los ítems que componen la escala, el siguiente paso consistió en calcular los coeficientes de consistencia interna, medidos a través de la prueba de alfa de Cronbach de la escala total y de cada una de las sub-escalas. Se eliminaron los ítems 5 (dimensión Negativos) y 10 (dimensión Social) por valores CCIT-c inferiores a .30, quedando así formada por 30 ítems con un alfa de .86, así como de las dimensiones Positivos (ítems 1, 2, 3 y 4) con alfa ,76; Negativos (ítems 6, 7 y 8) con alfa .67; Social (ítems 9, 11, 12, 21, 22, 23 y 24) alfa .76; Personal (ítems 13, 14, 15, 16, 17, 18, 19 y 20) alfa .70 y General (ítems $25,26,27,28,29$, 30, 31 y 32$)$ alfa .81 .

\section{Validez de constructo}

\section{Análisis Factorial Exploratorio (AFE)}

Siguiendo a Lloret-Segura, Ferreres-Traver, Hernández-Baeza y Tomás-Marco (2014), a pesar de la naturaleza politómica de los ítems, al contar con cinco alternativas de respuesta y seguir una distribución aproximadamente normal, se observó un rango de asimetría y curtosis en la mayoría de los ítems entre -2 y 2 (tabla 2) excepto para los ítems 4, 9, 11, $12,15,19,22,24,26,28,30$ y 31 . Además, se realizó un estudio de correlación mediante el índice de Pearson para comprobar la relación lineal entre las variables.

Como se observa en la tabla 3 , todas las subescalas correlacionaron entre ellas de forma significativa y la mayoría con un elevado grado de saturación. El siguiente paso fue estimar los factores de la escala, para lo cual se empleó el método denominado de máxima verosimilitud. Inicialmente se forzó la extracción a 5 factores que eran los esperados que existiesen con los 30 ítems seleccionados. Dado que no hubo ítems que se pudieran relacionar con una de las dimensiones, se optó por la extracción a 2 factores, atendiendo a la posibilidad de agruparlos en un factor centrado en la promoción de valores (que aglutinara las dimensiones de responsabilidad personal y social) otro factor vinculado con aspectos generales de satisfacción con la metodología implementada (dimensiones aspectos positivos y negativos de la nueva metodología y logros generales).

Tabla 2. Estadísticos descriptivos de los ítems.

\begin{tabular}{llllllllll}
\hline & $\mathrm{M}$ & $\mathrm{DT}$ & Asimetría & Curtosis & $\mathrm{M}$ & $\mathrm{DT}$ & Asimetría & Curtosis \\
\cline { 2 - 9 } Ítem 1 & 4.29 & 0.851 & -0.926 & 0.307 & Ítem 18 & 3.98 & 1.060 & -1.187 & 1.150 \\
Ítem 2 & 4.56 & 0.644 & -1.377 & 1.564 & Ítem 19 & 4.36 & 0.965 & -1.618 & 2.344 \\
Ítem 3 & 4.51 & 0.765 & -1.405 & 0.995 & Ítem 20 & 4.00 & 1.225 & -1.135 & 0.381 \\
Ítem 4 & 4.48 & 0.776 & -1.510 & 2.381 & Ítem 21 & 4.14 & 1.098 & -1.475 & 1.745 \\
Ítem 6 & 1.71 & 1.186 & 1.525 & 1.157 & Ítem 22 & 4.56 & 0.773 & -2.024 & 4.422 \\
Ítem 7 & 1.99 & 1.301 & 1.032 & -0.224 & Ítem 23 & 4.19 & 0.960 & -1.138 & 0.895 \\
Ítem 8 & 2.03 & 1.426 & 1.082 & -0.299 & Ítem 24 & 4.28 & 1.002 & -1.653 & 2.704 \\
Ítem 9 & 4.32 & 0.985 & -1.752 & 3.158 & Ítem 25 & 4.29 & 0.926 & -1.315 & 1.489 \\
Ítem 11 & 4.53 & 0.817 & -2.097 & 5.010 & Ítem 26 & 4.46 & 0.866 & -1.760 & 3.153 \\
Ítem 12 & 4.74 & 0.602 & -2.374 & 5.201 & Ítem 27 & 4.12 & 0.871 & -0.936 & 1.128 \\
Ítem 13 & 4.49 & 0.685 & -1.143 & 0.688 & Ítem 28 & 4.36 & 0.866 & -1.487 & 2.442 \\
Ítem 14 & 4.38 & 0.878 & -1.204 & 0.365 & Ítem 29 & 4.23 & 0.955 & -1.356 & 1.778 \\
Ítem 15 & 4.36 & 0.855 & -1.735 & 4.014 & Ítem 30 & 4.61 & 0.700 & -2.263 & 6.537 \\
Ítem 16 & 4.06 & 1.273 & -1.119 & 0.049 & Ítem 31 & 4.50 & 0.776 & -1.673 & 3.087 \\
Ítem 17 & 3.98 & 1.207 & -1.049 & 0.225 & Ítem 32 & 4.51 & 0.708 & -1.117 & -0.127 \\
\hline
\end{tabular}




\section{Rodríguez, I.; Valero-Valenzuela, A.; Manzano-Sánchez, D.}

Tabla 3. Estadísticos descriptivos y correlaciones bivariadas de las sub-escalas.

\begin{tabular}{lllllllll}
\hline & Rango & M & DT & Positivos & Negativos & Social & Personal & General \\
\cline { 2 - 8 } Positivos & $1-5$ & 4.46 & 0.58 & - & $-.236^{* *}$ & $.412^{* *}$ & $.476^{* *}$ & $.427^{* *}$ \\
Negativos & $1-5$ & 1.91 & 1.02 & - & - & $-.253^{* *}$ & $-.289^{* *}$ & $-.277^{* *}$ \\
Social & $1-5$ & 4.39 & 0.58 & - & - & - & $.518^{* *}$ & $.550^{* *}$ \\
Personal & $1-5$ & 4.20 & 0.59 & - & - & - & - & $.502^{* *}$ \\
General & $1-5$ & 4.39 & 0.55 & - & - & - & - & - \\
\hline
\end{tabular}

Nota: **. La correlación es significativa al nivel .01 (bilateral).

Como método para la asignación de ítems a cada factor se seleccionó la rotación oblicua Oblimin directo y como índices de la muestra se obtuvo el KMO y la prueba de esfericidad de Bartlett. El índice KMO fue de .871 , lo que indica la adecuación de los datos al AFE "satisfactoria" (Lloret-Segura et al., 2014). En la prueba de esfericidad de Bartlett se obtuvo una p-valor de .000 , lo que constató la existencia de una alta correlación entre las variables.

Debido a que algunos de los ítems 32 no saturaban con el mínimo establecido de .400 (Lloret-Segura et al., 2014), se procedió a eliminar del análisis y a realizar un nuevo análisis factorial con la escala reducida tras eliminar estos ítems (tabla 4), conformándose la escala con 12 ítems divididos en dos factores: promoción de valores (6 ítems) y satisfacción con la metodología (6 ítems).

Tabla 4. Análisis Factorial Exploratorio (12 ítems).

\begin{tabular}{lcc}
\hline Ítem & $\begin{array}{c}\text { Factor 1. Promoción de } \\
\text { valores }\end{array}$ & $\begin{array}{c}\text { Factor 2. Aspectos } \\
\text { generales del modelo }\end{array}$ \\
\hline Ítem 3 & & .562 \\
Ítem 9 & .535 & \\
Ítem 12 & .573 & \\
Ítem 14 & .506 & \\
Ítem 16 & .497 & \\
Ítem 19 & .690 & \\
Ítem 24 & .622 & .725 \\
Ítem 25 & & .695 \\
Ítem 26 & & .666 \\
Ítem 29 & & .542 \\
Ítem 30 & & .790 \\
Ítem 31 & & \\
\hline
\end{tabular}

Análisis Factorial Confirmatorio (AFC).

Se aplicaron modelos de ecuaciones estructurales, evaluando la estructura factorial del instrumento mediante AFC utilizando el paquete "SEM" del programa estadístico R ( $\mathrm{R}$ Core Team, 2020), hipotetizando un modelo de medida consistente en 2 factores correlacionados, obteniendo una serie de parámetros a través del método de Máxima
Verosimilitud. Como recomiendan, entre otros autores, Bentler (2007), Markland (2007) o Miles y Shevlin (2007), se deben calcular varios índices de ajuste, por lo que se combinaron índices absolutos y relativos. La estructura factorial de la escala se obtuvo utilizando la matriz de covarianzas como método de entrada para el análisis de los datos y el método de estimación de máxima verosimilitud. Se realizó el AFC con los 12 ítems con los que se encontró explicación en el AFE, donde los índices ofrecían valores óptimos con 12 ítems, tal y como se detalla en la figura 2.

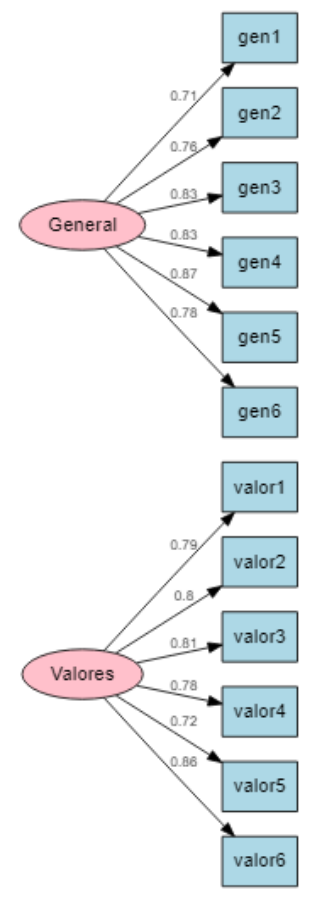

Figura 2. Path Diagram del AFC, con pesos estandarizados de cada uno de los ítems del Cuestionario ECVA-12 (Elaboración propia).

Los índices de bondad de ajuste obtenidos en el modelo de 12 ítems mostraron un ajuste satisfactorio 


\section{Medida de la Responsabilidad Personal y Social en contexto escolar}

$\left(\mathrm{X}^{2}=86.231(50) ; \mathrm{SRMR}=.043 ; \mathrm{TFI}=.934 ; \mathrm{CFI}\right.$ $=.950)$, excepto para el RMSEA con un valor ligeramente por encima de .08 (RMSEA $=.098[90 \%$ $\mathrm{CI}=0.062,0.133])$. Los pesos de regresión estandarizados de los ítems oscilaron entre .71 y .86 siendo todos ellos estadísticamente significativos, y la covarianza entre factores asume un valor positivo de .99 .

\section{Fiabilidad}

Se calculó el alfa de Cronbach de la escala total y de las 2 sub-escalas obtenidas a través del AFE. Se obtuvo un valor positivo (.856) atendiendo a los mínimos establecidos para la presente investigación y superior al obtenido con el modelo de 32 ítems (.846). Se evaluó la estabilidad temporal a través de la prueba test-retest, siguiendo trabajos como el de MerinoBarrero, Valero-Valenzuela y Moreno-Murcia (2017). La prueba mostró una correlación de .766, considerándolo un valor aceptable de correlación entre ambas respuestas (Vincent, 1995) y garantizando así la estabilidad temporal del instrumento.

\section{DISCUSIÓN}

El objetivo principal del presente estudio ha sido diseñar y validar un cuestionario que permita comparar la metodología previa empleada por el docente con el MRPS, midiendo el grado de satisfacción de los estudiantes y los cambios percibidos en su comportamiento, especialmente en los valores adquiridos con su implementación.

Se ha realizado un proceso de validación y fiabilidad con diferentes procedimientos como la validación de contenido mediante jueces expertos, la validez de comprensión y la fiabilidad de la escala, siguiendo procedimientos similares a los empleados en oras investigaciones (Ortega et al., 2009).

El análisis factorial exploratorio mostró un índice KMO satisfactorio y la eliminación de los ítems que no saturaban con el mínimo establecido conformó una escala dividida en dos factores con 12 ítems en total, por lo que la versión final del cuestionario analizado presenta una validez de constructo sólida. Además, los valores de alfa de Cronbach son superiores al mínimo establecido por Nunnally (1978). Las diferentes subescalas presentaron correlaciones significativas entre todas ellas asegurando la homogeneidad de cada una de las dimensiones (Carretero-Dios y Pérez, 2005) y la estabilidad temporal supera los valores establecidos por Vincent (1995). Todos estos análisis descritos señalan que el instrumento tiene unas adecuadas propiedades psicométricas.

En el AFC se obtuvieron índices robustos que favorecían un buen ajuste del modelo, excepto para el RMSEA. De este modo, la estructura definitiva de la escala comparativa entre la metodología anteriormente utilizada por el profesor y el MRPS se compone de 12 ítems distribuidos en 2 factores diferentes: Aspectos generales y Aspectos específicos personales y sociales.

Una revisión de la literatura científica existente muestra que no hay un instrumento como el que se presenta en esta investigación. Se han desarrollado instrumentos para medir aspectos concretos de la responsabilidad en el aula incluyendo el entorno familiar (Monsalvo, 2012) y otros que valoran el nivel de responsabilidad personal y social de los estudiantes (Escartí, et al., 2015), la progresión alcanzada aplicando el modelo de forma observacional (Camerino et al., 2020) y el clima generado en el aula de Educación Física (Fernández-Río et al., 2019), además de valorarse aspectos relacionados con el MRPS mediante análisis de entrevistas (ManzanoSánchez et al., 2021). Sin embargo, no existe un instrumento de medida que evalúe los cambios en el comportamiento del estudiante tras la aplicación del modelo, ni tampoco que tenga en cuenta no solo la materia de Educación Física, sino el resto de las materias curriculares.

Por lo tanto se trata de una importante aportación al desarrollo del MRPS y a la literatura relacionada con él y especialmente para poder ver si realmente este modelo sirve para el fomento de valores educativos además de la responsabilidad. Además, este cuestionario puede servir para valorar otras metodologías que busquen el fomento de valores educativos, ya que todos los ítems se podrían utilizar, siendo únicamente el ítem 6 ("Sea capaz de autoevaluarme y de evaluar a mi maestro/profesor y a mis compañeros") más específico del MRPS, pero que se sugiere podría ser incluido en cualquier clase, dada la importancia de la evaluación personal.

Si bien, son varias las limitaciones que se encuentran en este estudio. Por un lado, el instrumento sólo se pudo validar con grupos en los que el docente estaba poniendo en práctica el MRPS y es difícil encontrar profesionales de la educación que conozcan y estén aplicándolo en sus aulas. Además, no todos los jueces expertos se implicaron de igual forma, pues varios de 


\section{Rodríguez, I.; Valero-Valenzuela, A.; Manzano-Sánchez, D.}

ellos no hicieron aportaciones a nivel cualitativo, lo que podía haber afinado más el diseño del cuestionario. En otro orden, la edad de los participantes fue similar, pero no la misma para los dos análisis realizados ( 9 a 15 años y 12 a 15 años), lo cual, podría haber influido en los resultados.

Por otro lado, una de las grandes limitaciones ha sido la muestra de estudiantes participantes, ya que han sido seleccionados por conveniencia y con un bajo tamaño muestral. Esto ha sido debido a que el universo realmente es escaso, puesto que la población que únicamente se pueden considerar para formar parte de esta investigación, son estudiantes que hayan recibido o estén recibiendo esta metodología de enseñanza, lo cual limita en gran medida la accesibilidad a una muestra que cumpla con esta característica. Finalmente, los valores de RMSEA obtenidos en el cuestionario fueron altos pero no los considerados como ideales, siendo a su vez interesante. realizar un nuevo estudio que pudiera abordar la validez de criterio del presente cuestionario permitiendo arrojar una puntuación global en la utilización del cuestionario.

\section{CONCLUSIONES}

Atendiendo al principal objetivo de la investigación y a los resultados obtenidos, se confirma que el instrumento diseñado cumple con los criterios de validez y fiabilidad establecidos. Se trata de una herramienta válida y fiable que permite al docente conocer los cambios y logros adquiridos por el alumnado gracias a la implementación del MRPS, comparando ciertas conductas y actitudes con la metodología aplicada anteriormente.

El instrumento validado se convierte en una herramienta que puede permitir al docente conocer cómo y cuánto funciona el MRPS con su grupo de alumnos, pudiendo comprobar su utilidad y apreciar las ventajas desde la perspectiva del alumnado.

La escala comparativa de los valores adquiridos con el MRPS frente a la metodología anterior en escolares (ECVA-12) (anexo) permite conocer los valores y las conductas que el alumnado logra tras la aplicación del MRPS en el aula y en comparación con la metodología utilizada anteriormente por su profesor, pudiendo comprobar los cambios que dicho modelo genera en los estudiantes y la satisfacción que genera entre los estudiantes.

\section{REFERENCIAS (APA $7^{\text {a }}$ EDICION)}

1. Albaladejo-Blázquez, N., Ferrer-Cascales, R., Reig-Ferrer, A., Fernández-Pascual, M. (2013). Does School Violence occur in pre-school and primary education? A proposal for assessment and management. Anales De Psicología, 29(3), 10601069 org/10.6018/analesps.29.3.158431

https://doi.

2. Ayala-Carrillo, M. (2015). Violencia escolar: un problema complejo. Ra Ximhai, 11(4), 493-509. https://bit.ly/3v6uFPd

3. Bentler, P. M. (2007). On tests and indices for evaluating structural models. Personality and Individual Differences, 42, 815-829. https://doi.org/10.1016/J.PAID.2006.09.024

4. Byrne, B. (2010). Structural Equation Modeling with AMOS Basic Concepts, Applications, and Programming. New York, NY, USA: Taylor y Francis Group, LLC.

5. Camerino, O., Valero-Valenzuela, A., Prat, Q., Manzano-Sánchez, D., Castañer, M. (2019). Optimizing Education: A Mixed Methods Approach Oriented to Teaching Personal and Social Responsibility (TPSR). Frontiers in Psychology, $\quad 10, \quad 1-15$. https://10.3389/fpsyg.2019.01439.

6. Carreres-Ponsoda, F., Escartí, A., JimenezOlmedo, J. M., Cortell-Tormo, J. M. (2021). Effects of a Teaching Personal and Social Responsibility Model Intervention in Competitive Youth Sport. Frontiers in Psychology, 12, 408420. https://doi.org/10.3389/fpsyg.2021.624018

7. Carretero-Dios, H., Pérez, C. (2005). Normas para el desarrollo y revisión de estudios instrumentales. International Journal of Clinical and Health Psychology, 5, 521-551.

8. Escartí, A., Gutiérrez, M., Pascual, C. (2011). Propiedades psicométricas de la versión española del Cuestionario de Responsabilidad Personal y Social en contextos de Educación Física. Revista de Psicología del Deporte, 20, 119-130.

9. Escartí, A., Llopis-Goig, R., Wright, P. (2018). Assessing the Implementation Fidelity of a School-based Teaching Personal and Social Responsibility Program in Physical Education and Other Subject Areas. Journal of Teaching in Physical Education, 37, 12-23. https://doi.org/10.1123/jtpe.2016-0200 


\section{Medida de la Responsabilidad Personal y Social en contexto escolar}

10. Escartí, A., Wright, P. M., Pascual, C., Gutiérrez, M. (2015). Tool for Assessing Responsibilitybased Education (TARE) 2.0: Instrument revisions, inter-rater reliability, and correlations between observed teaching strategies and student behaviors. Universal Journal of Psychology, 3(2), 55-63. https://doi.org/10.13189/ujp.2015.030205

11. Fernández-Río, J., Cecchini, J. A., MerinoBarrero, J. A., Valero-Valenzuela, A. (2019). Perceived Classroom Responsibility Climate Questionnaire: A new scale. Psicothema, 31, 475481. https://doi.org/ 10.7334/psicothema2019.76.

12. Fernández, J., Méndez, A. (2016). El Aprendizaje Cooperativo: Modelo Pedagógico para Educación Física. Retos de la Actividad Física y el Deporte, 29, 201-206.

13. Gordon, B., Jacobs, J. M., Wright, P. M. (2016). Social and emotional learning through a teaching personal and social responsibility based afterschool program for disengaged middle-school boys. Journal of Teaching in Physical Education, 35(4), 358-369. https://doi. org/10.1123/jtpe.2016-0106

14. Hair, J., Black, W., Babin, B., Anderson, R. (2014). Multivariate Data Analysis. London, UK: Pearson Educational, Inc.

15. Hellison, D. (1985). Goal and strategies for teaching physical education. Champaign, IL: Human Kinetics.

16. Lloret-Segura, S., Ferreres-Traver, A., Hernández-Baeza, A., Tomás-Marco, I. (2014). El análisis factorial exploratorio de los ítems: una guía práctica, revisada y actualizada. Anales de Psicología, 30(3), 1151-1169. https://doi.org/10.6018/analesps.30.3.19936.

17. Manzano-Sánchez, D., Valero-Valenzuela, A. (2019). The personal and social responsibility model (TPSR) in the different subjects of primary education and its impact on responsibility, autonomy, motivation, self-concept and social climate. Journal of Sport and Health Research, 11, 273-288.

18. Manzano-Sánchez, D., Valero-Valenzuela, A. (2019). Implementation of a Model-Based Programme to Promote Personal and Social Responsibility and Its Effects on Motivation, Prosocial Behaviours, Violence and Classroom Climate in Primary and Secondary Education. International journal of environmental research and public health, 16(21), 1-17. https://doi.org/10.3390\%2Fijerph16214259

19. Manzano-Sánchez, D., González-Villora, S., Valero-Valenzuela, A. (2021). Application of teaching personal and social responsibility model to the Secondary Education curriculum. Implications for students and teachers. International Journal of Environmental and Public Health. 18(6), 3047, 1-14. https://doi.org/10.3390/ijerph18063047

20. Manzano-Sánchez, D., Conte-Marín, L., GómezLópez, M., Valero-Valenzuela, A. (2020). Applying the Personal and Social Responsibility Model as a School-Wide Project in All Participants: Teachers' Views. Frontiers in Psychology, $11 \quad$ (579), $1-8$. https://doi.org/10.3389/fpsyg.2020.00579

21. Markland, D. (2007). The golden rule is that there are no golden rules: A commentary on Paul Barret's recommendations for reporting model fit in structural equation modelling. Personality and Individual Differences, 42, 851-858. https://doi. org/10.1016/j.paid.2006.09.023

22. Marsh, H., Hau, K., Wen, Z. (2014). In search of golden rules: Comment on hypothesis testing approaches to setting cutoff values for fit indexes and dangers in overgeneralizing $\mathrm{Hu}$ and Bentler's (1999) findings. Structural Equation Modeling, 11 , 320-341. doi.org/10.1207/s15328007sem1103_2

23. Melero-Cañas D., Morales-Baños V., ManzanoSánchez D., Navarro-Ardoy D., ValeroValenzuela A. (2021) Effects of an Educational Hybrid Physical Education Program on Physical Fitness, Body Composition and Sedentary and Physical Activity Times in Adolescents: The Seneb's Enigma. Frontiers in psychology, 11, https://doi.org/10.3389/fpsyg.2020.629335

24. Menéndez-Santurio, J. I., Fernández Río, F. J. (2018). Actitudes hacia la violencia y papel importante del alumnado en el aula de educación física. Revista complutense de educación, 29(4), 1293-308. https://doi.org/10.5209/RCED.55352

25. Menéndez, J. I., Fernández, J. (2016). Violencia, responsabilidad, amistad y necesidades psicológicas básicas: efectos de un programa de Educación Deportiva y Responsabilidad Personal y Social. Revista de Psicodidáctica, 21(2), 245260.

https://doi.org/10.1387/RevPsicodidact.15269 


\section{Rodríguez, I.; Valero-Valenzuela, A.; Manzano-Sánchez, D.}

26. Merino, C., Livia, S. (2009). Intervalos de confianza asimétricos para el índice de la validez de contenido: un programa Visual Basic para la $\mathrm{V}$ de Aiken. Anales de Psicología, 25 (1), 169-171. https://bit.ly/3naEzML

27. Merino-Barrero, J.A., Valero-Valenzuela, A., Moreno-Murcia, J.A. (2017). Análisis psicométrico del cuestionario estilos de enseñanza en Educación Física (EEEF). Revista Internacional de Medicina y Ciencias de la Actividad Física y el Deporte, 17 (66), 225-241. https://doi. org/10.15366/rimcafd2017.66.002

28. Miles, J., Shevlin, M. (2007). A time and a place for incremental fit indices. Personality and Individual Differences, 42(5), 869-874. https://doi. org/10.1016/j.paid.2006.09.022

29. Monsalvo, E. (2012). Escala de Evaluación de las Actitudes de Responsabilidad Social en Alumnos de Educación Primaria (EARSA-P). Departamento de Psicología, Universidad de Valladolid, España.

30. Nunnally, J. C. (1978). Psychometric theory. New York: McGraw-Hill.

31. Ortega, E., Calderón, A., Palao, J., Puigcerver, M. (2009). Diseño y validación de contenido de un cuestionario sobre la satisfacción, participación y opinión de mejora en las clases de Educación Física en secundaria. Wanceulen E.F. Digital, 5, 14-26.

32. Osterlind, S.J. (1989). Constructing test items. Boston: Kluwer.

33. Pan, Y. H., Keh, N. C. (2014). Teaching Responsibility Through Physical Education: Research and Applications in Taiwan. スポー少教育学研究, 34(1)， 63-69. https://doi.org/10.7219/JJSES.34.1_63

34. $\mathrm{R}$ Core Team (2020). $R$ : A language and environment for statistical computing. $R$ Foundation for Statistical Computing. Viena, Austria. https://bit.ly/3ekZEQF

35. Reguant-Álvarez, M., Torrado-Fonseca, M. (2016). El método Delphi. REIRE, Revista d'Innovació i Recerca en Educació, 9 (1), 87-102. https://doi.org/10.1016/0040-1625(91)90040-M.

36. Ruiz, L. M., Rodríguez, P., Martinek, T., Schilling, T., Durán, L. J., Jiménez, P. (2006). El proyecto esfuerzo: un modelo para el desarrollo de la responsabilidad personal y social a través del deporte. Revista de Educación, 341, 933-958.
37. Sánchez-Alcaraz, B. J., Gómez-Mármol, A., Valero-Valenzuela, A., De la Cruz, E., DíazSuárez, A. (2016). El Modelo de Responsabilidad Personal y Social a través del deporte como propuesta metodológica para la educación en valores en adolescentes. Espiral. Cuadernos del Profesorado, 9 (18), $16-26$. https://doi.org/10.47197/retos.v37i37.67890

38. Vincent, J. W. (1995). Statistics in kinesiology. Champaign, IL: Human Kinetics.

39. Wright, P. M., Craig, M. W. (2011). Tool for Assessing Responsibility-Based Education (TARE): Instrument development, content validity, and interrater reliability. Measurement in Physical Education and Exercise Science, 15(3), 204-219. https://doi. org/10.1080/1091367X.2011.590084

40. Yagüe, J., Herrero, A., Tabernero, B., Veroz, R. (2017). Diseño y validación del cuestionario "Deporteduca" para conocer la incidencia de la competición sobre algunos valores socioeducativos en el ámbito de la iniciación al fútbol. Retos: nuevas tendencias en educación física, deporte y recreación, 31, 197-201. https://doi.org/10.47197/retos.v0i31.51391 


\section{Anexo. \\ Escala Comparativa de los Valores Adquiridos con el Modelo de Responsabilidad Personal y Social (ECVA-12)}

En comparación con la anterior forma de impartir las Valoración

clases de mi maestro/profesor, el Modelo de

Responsabilidad ha hecho que...

Valora de 1 (muy en desacuerdo) a 5 (muy de acuerdo)

las siguientes cuestiones.

1. Me relacione mejor con mis compañeros y con el profesor

2. Experimente un mejor clima de respeto en clase

3. Respeto y cuide los materiales

4. Participe más en las clases

5. Le dé más importancia a mi mejora personal en lugar de fijarme en los demás

6. Sea capaz de autoevaluarme y de evaluar a mi maestro/profesor y a mis compañeros (técnica del pulgar)

7. Sea capaz de organizar una tarea y explicarla a mis compañeros

8. Aplique lo que he aprendido en clase a situaciones fuera del aula como, por ejemplo, organizar mis tareas en casa

9. Adquiera valores como respetar y ayudar a los demás. Por ejemplo, jugando a deportes de equipo en la calle, ayudando a personas mayores cuando lo necesitan, ...

10. Aprenda a mediar para ayudar a resolver posibles problemas entre compañeros

11. El profesor nos enseñe cómo ser más responsables a través de los contenidos de la asignatura

12. Me sienta más seguro de mí mismo a la hora de realizar las tareas 